\title{
Amorphization of Indium Phosphide Single Crystals by Swift Heavy Ion Bombardment
}

\author{
A.S. Khalil
}

Mining and Metallurgy Department, Tabbin Institute for Metallurgical Studies (TIMS), Helwan, POB 109, Cairo, Egypt

Irradiation of single crystalline compound semiconductors with energetic heavy ion beams produces disorder in the irradiated crystal lattices. Indium Phosphide (InP) is an important technological III-V compound semiconductor used in space solar arrays and a variety of electronic devices.

In this investigation, electron transparent and bulk samples cut from InP (001) wafer were bombarded by $200 \mathrm{MeV} \mathrm{Au}{ }^{+}$ions $(\sim 1 \mathrm{MeV} / \mathrm{amu})$ at fluencies ranging from $5 \times 10^{10}$ to $1 \times 10^{14} \mathrm{ion} / \mathrm{cm}^{2}$ in order to study the amorphization process of the InP (complete atomic disorder of the original InP crystal lattice).

As shown in figures 1(a) to 1(c) for thin foil samples investigated by TEM, the progression of amorphization is followed with increasing the fluence of irradiating $\mathrm{Au}^{+}$ions. Complete amorphization occurs at fluence of $>5 \times 10^{13}$ as confirmed by the inset showing the selected area electron diffraction pattern were the diffused rings and the absence of any diffraction spots are synonymous with amorphous state. This observation was further supplemented by Rutherford Backscattering Spectrometry (RBS/C technique) for the bulk InP samples which showed that at irradiation fluence of $1 \times 10^{14}$ ion $/ \mathrm{cm}^{2}$ the sample surface was completely amorphous.

Generally, amorphization in swift heavy ion irradiated compound semiconductors like InP can best be described by a combination of both heterogeneous mechanism (direct impact amorphization were each ion creates an amorphous ion track) and homogenous mechanism (defect accumulation were each ion track consists of an agglomeration of point defects). Models have been developed wherein an impinging ion can produce both a combination and coexistence of both amorphous-ion tracks and point defects-ion tracks which, when these ion tracks accumulate and overlap, the crystal converts to amorphous state [1].

By invoking the Hecking model [2] for the accumulation of damage we were able to show that the amorphization process proceeds by the accumulation and overlap of individual ion tracks. An Indicator of the amorphization process are the values of relative disorder $(\Delta \chi)_{\min }$ defined according to the following formula:

$$
\Delta(\chi)_{\min }=\frac{Y_{\text {irradiated }}-Y_{\text {Uniradiated }}}{Y_{\text {random }}-Y_{\text {Unirradiated }}}, \text { here } Y \text { is the yield from the RBS/C measurements. }
$$

The value $(\Delta \chi)_{\min }$ gives an estimate of the relative amount of disorder in the crystal and it reaches unity for a complete amorphous state. Plotting $(\Delta \chi)_{\min }$ versus the $\mathrm{Au}^{+}$ion fluence as depicted in figure 2 we conclude that the progression of amorphization might favor the homogenous mechanism [3]. This was further confirmed by HRTEM observations on ion track cores which indicate that individual ion track cores are not amorphous as shown in figure 3. 


\section{References:}

[1] W. J. Weber, Instruments and Methods B, 166/167 (2000), p. 98.

[2] N. Hecking, K. Heidemann and E. Kaat, Nuclear Instruments and Methods B, 15 (1986), p. 760.

[3] J. Nord, K. Nordlund and J. Keinonen, Physical Review B 65 (2002), article number 165329.

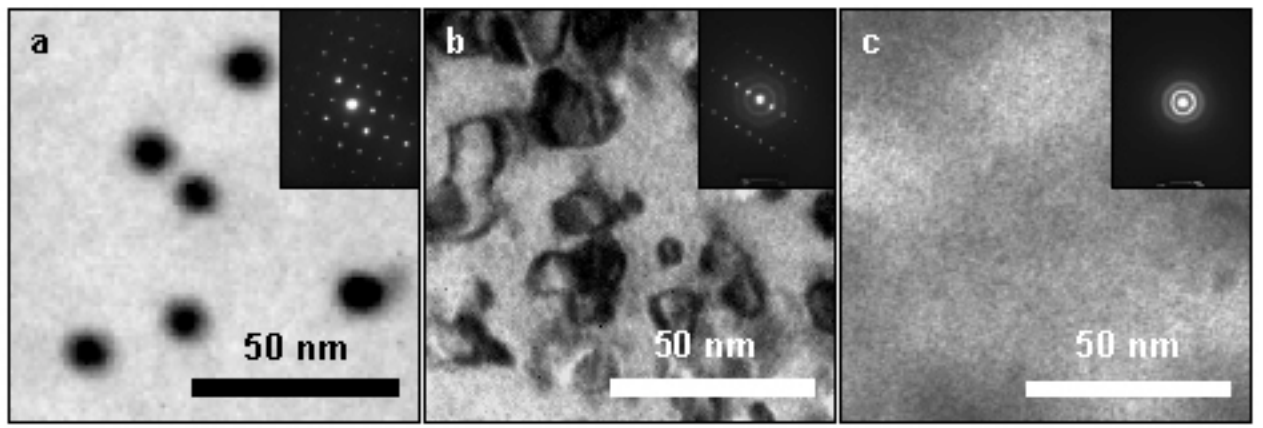

Figure 1. TEM micrographs for three different irradiating fluencies. In (a) for $5 \times 10^{10}$ ion $/ \mathrm{cm}^{2}$, the ion tracks are well separated and in (b) overlap leads to amorphous pockets at $1 \times 10^{13} \mathrm{ion} / \mathrm{cm}^{2}$. These will grow with increasing ion fluencies till complete amorphization occurs at fluence of $1 \times 10^{14}$ ion $/ \mathrm{cm}^{2}$ shown in (c).

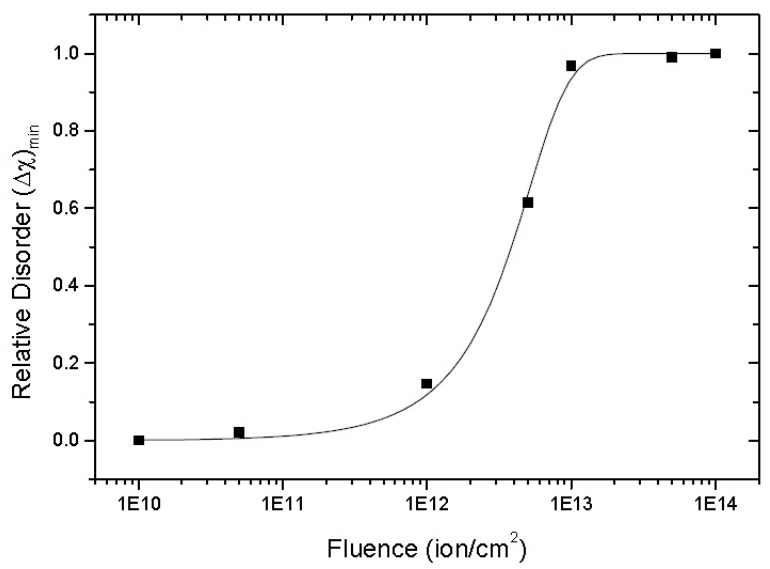

Figure 2. The plot of $(\Delta \chi)_{\min }$ versus ion fluencies, the best fit can be described by the composite Hecking model which gives more weight to homogenous amorphization.

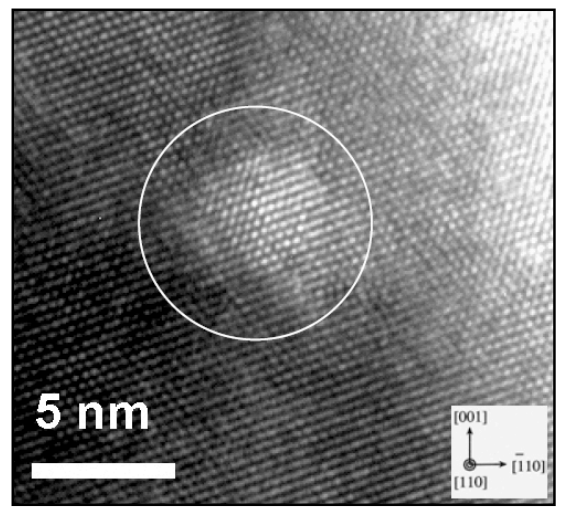

Figure 3. HRTEM micrograph of an ion track core, the continuation of lattice fringes implies that the ion track is not amorphous. 